\title{
A review of cloud security based on cryptographic mechanisms
}

\begin{abstract}
Recently, computer users can maintain their data and applications on the Internet and in the central remote server by using a technology called cloud computing. It is a kind of technology that allows users to access their personal files from any device provided with Internet services such technology offers even more applications to be utilized without installation. This paper presents a review of basic security challenges consisting of traditional security issues. It also discusses the additional challenges that take place as a result of using cloud computing model by providers and users of cloud systems. Furthermore, solutions suggested by some researchers are presented with a focus on cryptographic techniques that approve secure cloud storage.
\end{abstract}

Keyword: Cloud computing; Cryptographic techniques; Multicast; Symmetric algorithms 